\title{
Invasive Solid Papillary Carcinoma of Male Breast: A Rare Case Report
}

\author{
Arzu Ozsoy, ${ }^{1,}$ Nurdan Barca, ${ }^{1}$ Betul Akdal, ${ }^{1}$ Serra Kayacetin, ${ }^{2}$ and Levent Araz ${ }^{1}$ \\ ${ }^{1}$ Department of Radiology, Ankara Numune Training and Research Hospital, Ankara, Turkey \\ ${ }^{2}$ Department of Pathology, Ankara Numune Training and Research Hospital, Ankara, Turkey \\ "Corresponding author: Arzu Ozsoy, Department of Radiology, Ankara Numune Training and Research Hospital, Ankara, Turkey. Tel: +90-3125954863, Fax: +90-3124953475, \\ E-mail: arzu.ozsoy@hotmail.com
}

Received 2016 June 07; Revised 2016 December 16; Accepted 2017 February 15.

\begin{abstract}
Invasive papillary carcinoma of the breast is a rare form of breast carcinoma. Hereby we report a case of invasive papillary carcinoma of the breast in a 76-year-old otherwise healthy male, who presented at our department with the complaint of left subareolar masses. Mammography demonstrated two masses that were oval and round shaped, sharply demarcated and with micro calcifications on the internal structure. Ultrasonographic (US) analysis revealed solid mass lesions in the left subareolar location. These lesions had cystic areas in the interior structures. Color Doppler US revealed a high resistant vascularization in the central and peripheral parts of the lesions. Pathological examination of the specimen revealed the intracystic component to be invasive papillary carcinoma. In focal areas there was also findings of neuroendocrine differentiation. The patient underwent total mastectomy following an excisional biopsy, as the surgical margin after biopsy was positive for carcinoma. Axillary dissections had negative lymph nodes. After adjuvant chemotherapy and at the 2-year follow-up examination there were no recurrences or metastases. A high index of suspicion is necessary in male patients with solid lesions with cystic components.
\end{abstract}

Keywords: Male, Breast Cancer, Papillary Carcinoma

\section{Introduction}

Male breast cancers accounts for less than $1 \%$ of all breast cancer cases. Recent epidemiological studies have indicated that the male breast cancer incidence is increasing by $1.1 \%$ every year $(1,2)$.

The average age at diagnosis for men with breast cancer is 67 years, which is about 5 - 10 years older than the average age at diagnosis for women $(1,3)$. The most common clinical finding is a painless mass localized in the subareolar region. Other symptoms include nipple ulceration or retraction, nipple discharge, skin thickening, axillary lymphadenopathy and gynecomastia.

The most common type of male breast cancer is invasive ductal carcinoma (IDC) (85\% - 95\%) (4-6). Invasive papillary carcinoma is a rare histological type seen at the rate of $2 \%$. Histologically, papillary carcinoma may be intraductal or intracystic and each type can be non-invasive or invasive, a distinction only possible through histopathological examination.

The aim of this study was to present the radiological findings of invasive solid papillary cancer with neuroendocrine differentiation, which is a rare histological subtype of intracystic papillary cancer in a male patient.

\section{Case Presentation}

A 76-year-old male presented with complaints of a mass in the left breast for three months. On physical examination, two fixed, hard masses approximately $2 \mathrm{~cm}$ in diameter were palpated under the areola. There was nipple retraction. There were no palpable axillary lymphadenopathies. There was no significant family medical history. The patient did not report any trauma to the breasts, nipple discharge, skin rash or fever. Bilateral mammography and ultrasonography were performed.

In the bilateral mammographic examination, two dense masses were observed with left subareolar localization. These masses were oval and round shaped, sharply demarcated and had micro calcifications on the internal structure. There was a slight thickening and retraction of the skin (Figure $1 \mathrm{~A}$ and $1 \mathrm{~B}$ ). Ultrasonographic (US) analysis revealed $22 \times 17 \mathrm{~mm}$ and $15 \times 10 \mathrm{~mm}$ solid mass lesions in the left subareolar localization. They were oval and round shaped, sharply demarcated and had microlobulations in contour. The lesions had cystic areas in the interior structures (Figure 2A and 2B). Color Doppler US revealed a high resistant vascularization in the internal and periphery of the lesions (Figure 3). There were no axillary pathologies. The lesions were reported as breast imaging - reporting and data system (BI-RADS) 4C and tissue diagnosis was suggested. After total excision of the lesion, the pathology diagnosis was reported as invasive papillary carcinoma with 
neuroendocrine differentiation.

Macroscopically, the excised material was $3 \times 2 \times 1.5$ $\mathrm{cm}$ in size, irregular shaped and of a dirty-white color. Microscopically, there were papillary structures filling the cystic areas (Figure 4). These structures were composed of hyperchromatic, atypical ductal epithelial cells with slightly irregular nuclear contours. Invasion of solid structures around the tumor was observed (Figure 5).

According to the modified Bloom Richardson, the lesion was reported as high grade (score: 9; tubule formation: 3; nuclear pleomorphism: 3; mitosis: 3; grade: 3).

Immunohistochemically, strong nuclear staining of estrogen and progesterone receptors in the tumor cells was detected. In focal areas with neuroendocrine differentiation, there was positivity with neuron specific enolase and synaptophysin (Figure 6). Chromogranin staining was negative. With these findings, the case was histopathologically diagnosed as solid papillary carcinoma with neuroendocrine differentiation.

As a result of the diagnosis of invasive carcinoma and the positive surgical margins during excision, mastectomy was planned. The sentinel lymph node on frozen section was free of tumor and was reported to be reactive lymph node. Therefore, simple mastectomy was performed. Tumor was detected in samples from the nipple, but epidermal involvement was not observed. It was decided that the patient was at stage T2NOMO and chemotherapy (4 cures of endoxan and epirubicin) and tamoxifen was used. At the 2year follow-up examination, the patient was in a stable condition and there were no recurrences or metastases.

\section{Discussion}

Male breast cancer is an uncommon disease, and it accounts for only $0.6 \%-1 \%$ of all breast cancer cases (7). The etiology of male breast cancer is unclear, but hormonal levels and testicular abnormalities play a role in the development of this disease. BRCA2 mutations have been shown to confer a significant risk of breast cancer in men. Other recognized risk factors include radiation exposure, family history of breast cancer, Klinefelter syndrome and different benign breast conditions $(8,9)$. In the current case, there were no risk factors other than advanced age.

The majority of breast cancers in male patients are invasive ductal carcinoma, and papillary carcinoma is extremely rare $(3,10,11)$. Papillary carcinoma is histologically divided into five sub-groups: ductal carcinoma in-situ (DCIS) developed from intraductal papilloma, papillary DCIS, encapsulated (intracystic) papillary carcinoma, solid papillary carcinoma and invasive papillary carcinoma (12). In the most recent ( 4 th) edition of the WHO classification of breast tumors, invasive papillary carcinoma is relegated to the section of 'rare types' of invasive breast carcinoma (13). The invasive papillary subtype constitutes less than $2 \%$ of all breast cancers in women (12). Burga et al. performed a study on 759 male patients with breast cancers. The incidence of invasive papillary carcinoma in male patients was found to be two times more common than in female counterparts (14).

Papillary carcinoma may present as a single nodule in the subareolar region, but it can also present as multiple nodules extending to the periphery $(3,10-12)$. The most common clinical presentation is a palpable mass. Serosanginous discharge has been reported in $25 \%$ of patients $(4,15)$. The current patient had two palpable masses in the subareolar area. There was a slight thickening and retraction of the nipple, but there was no discharge.

Papillary carcinoma is usually seen on mammography as round or oval shaped lesions. Although usually with sharp margins, the lesions may also have obscure margins (15). Due to the minimal fibrotic reactions, spiculated contours are generally not expected (16). In papillary carcinomas, coarse heterogeneous or pleomorphic calcifications can be observed (16). On the other hand, as in the current case, punctate microcalcifications can be seen $(15,16)$. In ultrasound examination, papillary carcinomas can be seen as a hypoechoic solid mass or complex cystic masses. (15, $17,18)$ On Doppler images, typically large internal blood supply or feeder vessels are seen $(15,16)$. In the current case, two hypoechoic masses were detected, which were sharply demarcated, including cystic areas in the internal structure and on the color Doppler US, there were internal masses with high resistant vascularization.

Pathology is characterized by fibrovascular proliferation characterized by loss of myoepithelial cells within fibrovascular papilla. Also in some cases as in the current case, neuroendocrine differentiation could be observed (19). Synaptophysin is considered to be one of the most specific markers for neuroendocrine differentiation (13). Our case was synaptophysin positive indicating neuroendocrine differentiation (Figure 6).

Treatment of the disease varies according to staging and presence of hormone receptors. After lumpectomy or mastectomy, adjuvant chemotherapy, radiotherapy and hormone therapy can be added (20). In the current patient, after mastectomy, chemotherapy and hormone therapy were added because of receptor positivity.

Although in both sexes, breast cancers have similar behaviors at the same stages, male breast cancers may be diagnosed later than those in females (16), which leads to a worse prognosis. However, invasive papillary carcinoma grows more slowly than invasive ductal carcinoma and has a lower prevalence of axillary and distant metastases (4). Since the prognosis of invasive papillary carcinoma is bet- 

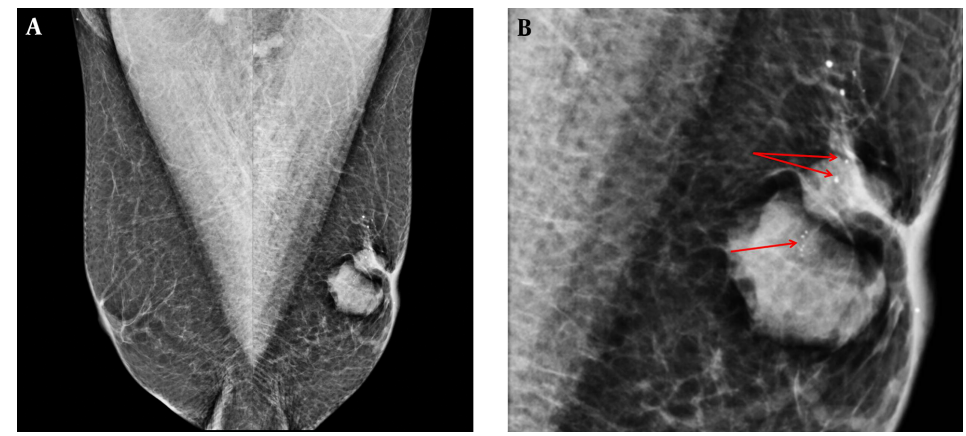

Figure 1. A 76-year-old man with a palpable left breast mass. A, Bilateral mediolateral oblique view (MLO) mammographic images. On the left MLO image, there were dense mass lesions in the retroareolar area that had irregular shapes, and microcalcifications. There was nipple retraction and thickening. B, Left MLO view magnified image. Rough heterogeneous microcalcification (red arrows) are seen.
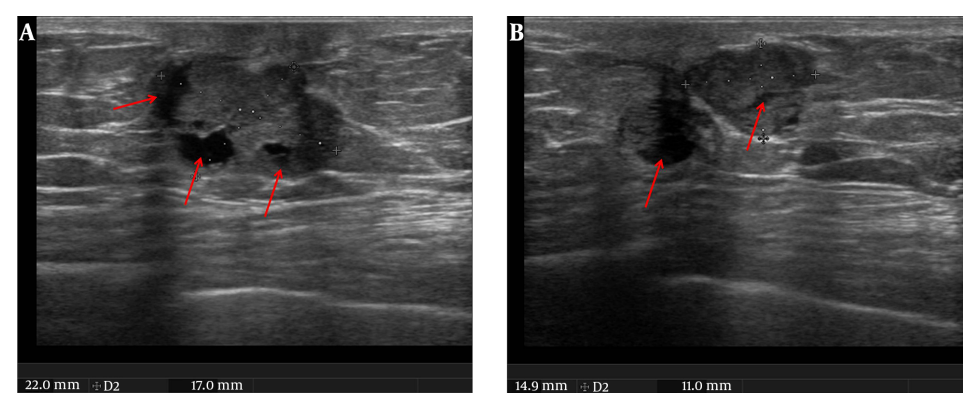

Figure 2. Ultrasound image in the same patient. A and B, Axial plane images. In the retroareolar area there were two irregular-shaped, well demarcated solid masses $(22 \times 17$ $\mathrm{mm}$ and $15 \times 10 \mathrm{~mm}$ in dimensions) with cystic areas (red arrows) in the interior part which had anti-parallel orientation to the skin.

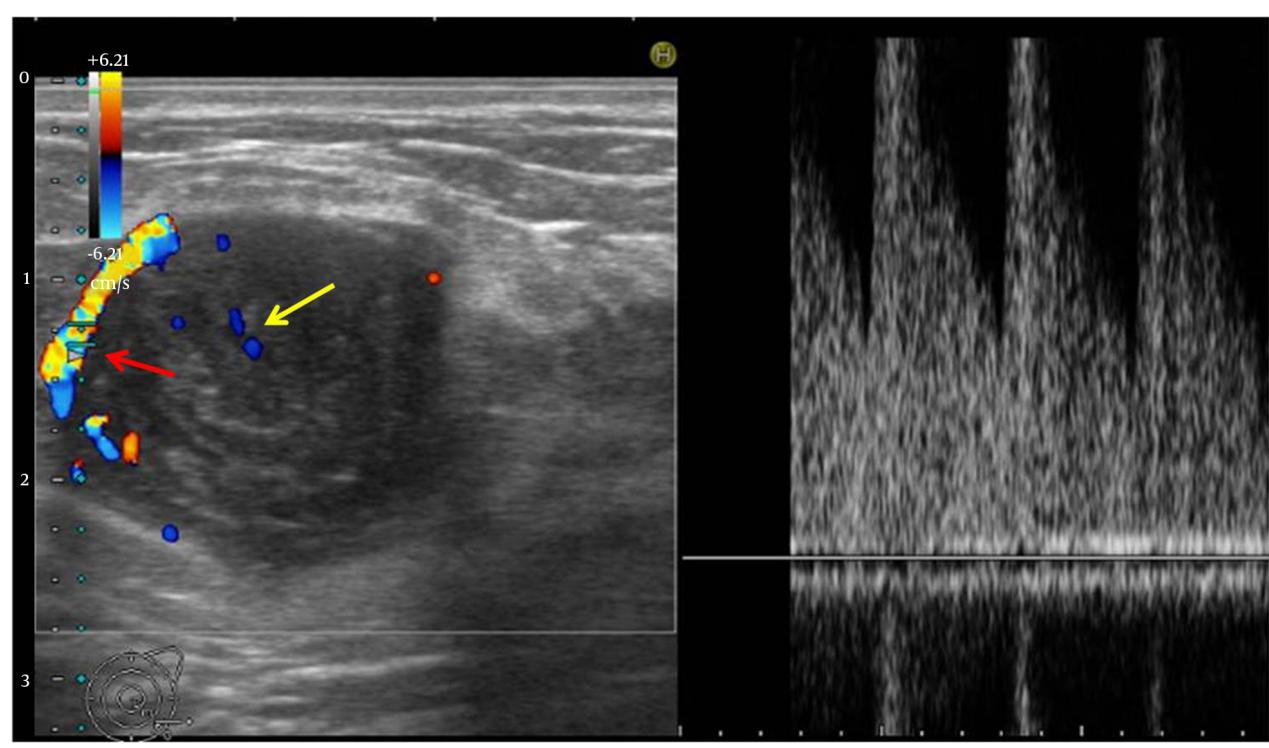

Figure 3. Color Doppler ultrasonography image. High resistant vascularization is seen in the central (yellow arrow) and periphery (red arrow) of the lesion. 


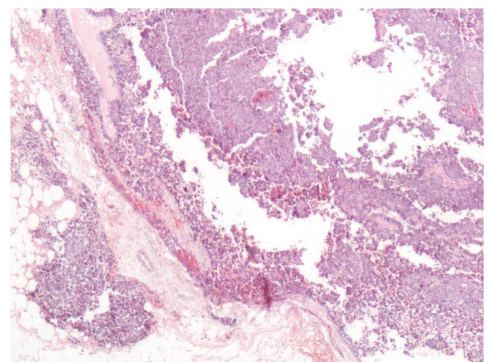

Figure 4. Papillary proliferations around the fibrovascular areas $(\times 100 ; \mathrm{H} \& \mathrm{E})$

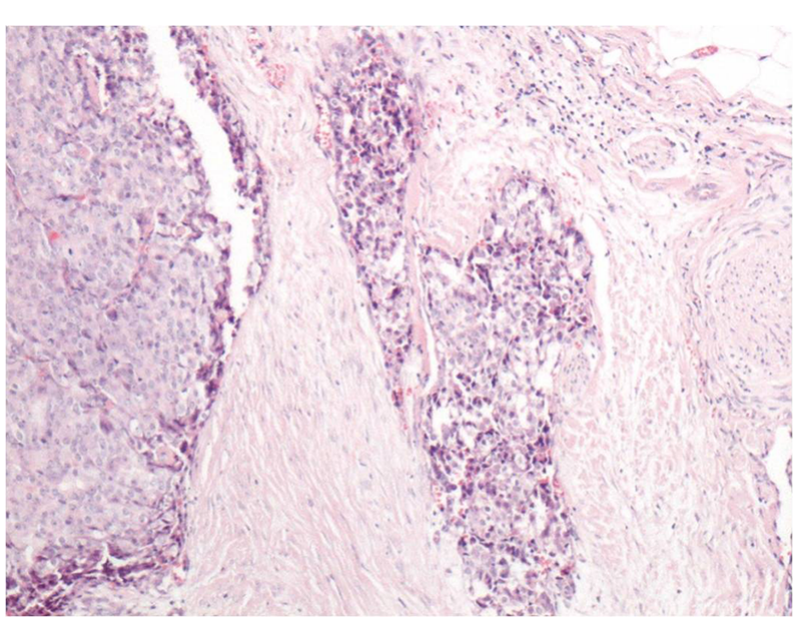

Figure 5. Solid structures indicating invasion around the tumor. $(\times 100 ; \mathrm{H} \& \mathrm{E})$

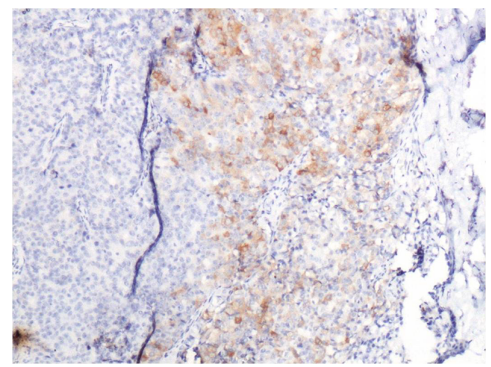

Figure 6. Focal positive staining with synaptophysin $(\times 400)$ ter than invasive ductal carcinoma, early diagnosis and treatment is important.

In conclusion, although invasive papillary carcinoma of the male breast is a rare pathology, a high index of suspicion is necessary in male patients with solid lesions with cystic components. Early diagnosis and treatment with mastectomy and adjuvant chemotherapy gives favorable results.

\section{Footnote}

Financial Disclosure: The authors have no financial interests related to the material in the manuscript.

\section{References}

1. Cutuli B, Le-Nir CC, Serin D, Kirova Y, Gaci Z, Lemanski C, et al Male breast cancer. Evolution of treatment and prognostic factors. Analysis of 489 cases. Crit Rev Oncol Hematol. 2010;73(3):246-54. doi: 10.1016/j.critrevonc.2009.04.002. [PubMed: 19442535].

2. Speirs V, Shaaban AM. The rising incidence of male breast cancer Breast Cancer Res Treat. 2009;115(2):429-30. doi: 10.1007/s10549-008 0053-y. [PubMed: 18478326].

3. Mathew J, Perkins GH, Stephens T, Middleton LP, Yang WT. Primary breast cancer in men: clinical, imaging, and pathologic findings in 57 patients. AJR Am J Roentgenol. 2008;191(6):1631-9. doi: 10.2214/AJR.08.1076. [PubMed:19020230].

4. Pant I, Joshi SC. Invasive papillary carcinoma of the male breast: report of a rare case and review of the literature. $J$ Cancer Res Ther. 2009;5(3):216-8. doi: 10.4103/0973-1482.57132. [PubMed: 19841568].

5. Haagensen CD. Diseases of the Breast. WB Saunders Company; 1971.

6. Giordano SH, Cohen DS, Buzdar AU, Perkins G, Hortobagyi GN. Breast carcinoma in men: a population-based study. Cancer. 2004;101(1):51-7. doi: 10.1002/cncr.20312. [PubMed: 15221988].

7. Giordano SH, Buzdar AU, Hortobagyi GN. Breast cancer in men. Ann Intern Med. 2002;137(8):678-87. [PubMed: 12379069].

8. Korde LA, Zujewski JA, Kamin L, Giordano S, Domchek S, Anderson WF, et al. Multidisciplinary meeting on male breast cancer: summary and research recommendations. J Clin Oncol. 2010;28(12):2114-22. doi 10.1200/JCO.2009.25.5729. [PubMed: 20308661].

9. Lakhani SR. WHO Classification of Tumours of the Breast. International Agency for Research on Cancer; 2012.

10. Grabowski J, Salzstein SL, Sadler GR, Blair S. Intracystic papillary carcinoma: a review of 917 cases. Cancer. 2008;113(5):916-20. doi: 10.1002/cncr.23723. [PubMed: 18661510].

11. Mulligan AM, O'Malley FP. Papillary lesions of the breast: a review. Adv Anat Pathol. 2007;14(2):108-19. doi: 10.1097/PAP.ob013e318032508d. [PubMed: 17471117].

12. Kline TS, Kannan V. Papillary carcinoma of the breast. A cytomorphologic analysis. Arch Pathol Lab Med. 1986;110(3):189-91. [PubMed: 3080975].

13. Tan PH, Schnitt SJ, van de Vijver MJ, Ellis IO, Lakhani SR. Papillary and neuroendocrine breast lesions: the WHO stance. Histopathology. 2015;66(6):761-70. doi: 10.1111/his.12463. [PubMed: 24845113].

14. Burga AM, Fadare O, Lininger RA, Tavassoli FA. Invasive carcinomas of the male breast: a morphologic study of the distribution of histologic subtypes and metastatic patterns in 778 cases. Virchows Arch. 2006;449(5):507-12. doi: 10.1007/s00428-006-0305-3. [PubMed: 17058095]. 
15. Schneider JA. Invasive papillary breast carcinoma: mammographic and sonographic appearance. Radiology. 1989;171(2):377-9. doi: 10.1148/radiology.171.2.2649917. [PubMed: 2649917].

16. Brookes MJ, Bourke AG. Radiological appearances of papillary breast lesions. Clin Radiol. 2008;63(11):1265-73. doi: 10.1016/j.crad.2008.02.012. [PubMed: 18929044].

17. Hu ZI, Liu C, Fisher PR, Cohen JA. Intracystic Papillary Carcinoma of the Breast in a Male Patient. Rare Tumors. 2016;8(1):6050. doi: 10.4081/rt.2016.6050. [PubMed: 27134706].

18. Sun Y, Howard-McNatt M. Intracystic papillary carcinoma of the breast in a male patient. Am Surg. 2014;80(3):E84-5. [PubMed: 24666852].

19. Pal SK, Lau SK, Kruper L, Nwoye U, Garberoglio C, Gupta RK, et al. Papillary carcinoma of the breast: an overview. Breast Cancer Res Treat. 2010;122(3):637-45. doi: 10.1007/s10549-010-0961-5. [PubMed: 20524058].

20. Fentiman IS, Fourquet A, Hortobagyi GN. Male breast cancer. Lancet. 2006;367(9510):595-604. doi: 10.1016/S0140-6736(06)68226-3. [PubMed: 16488803]. 